\title{
Does Acupuncture Have Any Benefit in the IVF Treatment of Couples with Unexplained Infertility; A Prospective Randomized Controlled Trial
}

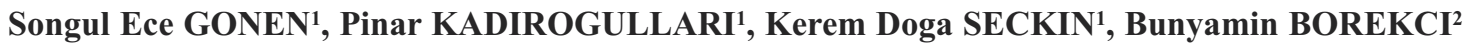 \\ Istanbul, Turkey
}

\begin{abstract}
OBJECTIVE: We aimed to investigate the effect of acupuncture performed as an adjuvant therapy to in vitro fertilization, on pregnancy rates by performing acupuncture on the day of embryo transfer, one hour before the procedure and one hour after the procedure.

STUDY DESIGN: In this open-label randomized prospective controlled study, 60 unexplained infertility patients enrolled for in vitro fertilization treatment were randomized by a computer-based number generator for acupuncture performance and no-treatment. All patients received $\mathrm{rFSH}\left(\mathrm{Gonal}-\mathrm{F}^{\circledR}\right)$ for ovulation induction and the antagonist Cetrorelix $\left(\right.$ Cetrotide $\left.^{\circledR}\right)$ to prevent premature ovulation. Ovulation was triggered by using hCG (Ovitrelle ${ }^{\circledR}$ ) and egg collection was done after $36-48$ hours. In the study group, acupuncture was performed by intradermal needling, bilaterally with a sterile needle, both one hour before and one hour after the embryo transfer. Acupuncture was not performed on the control group. The results of the treatment were evaluated 12 days after embryo transfer.
\end{abstract}

RESULTS: Thirty patients were randomized into the study group and 30 patients were randomly placed in the control group. Although there was no statistically significant difference between groups in terms of $\beta$-hCG positivity, it was $43.3 \%$ in the study group and $36.7 \%$ in the control group $(p>0.05)$.

CONCLUSION: The success rate of in vitro fertilization was higher in terms of pregnancy rate in the patient group who received acupuncture procedure as well, but the difference was not statistically significant. This may be due to the type of acupuncture procedure selected or insufficient sample size. As a result, further studies involving larger numbers of participants and using different acupuncture techniques are needed.

Keywords: Acupuncture, Infertility, In vitro fertilization

Gynecol Obstet Reprod Med 2020;26(1):38-43

\section{Introduction}

Infertility is defined as a failure to achieve pregnancy despite one year of unprotected sexual intercourse. (US Congress Office of Technology Assessment 1988) (1). Major causes of infertility are $35 \%$ male factor, $35 \%$ tubal and pelvic pathologies, $15 \%$ ovulation disorders, $10 \%$ unknown infertility, $5 \%$

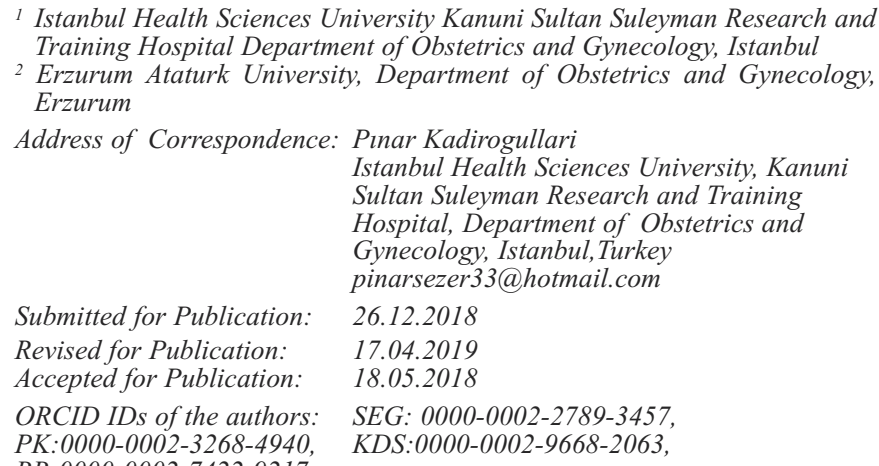

\begin{tabular}{|c|c|}
\hline $\begin{array}{c}\text { Quick Response Code: } \\
\text { 回 }\end{array}$ & Access this article online \\
\cline { 2 - 2 } & $\begin{array}{l}\text { Website: www.gorm.com.tr } \\
\text { e- mail: info@gorm.com.tr }\end{array}$ \\
\cline { 2 - 3 } & DOI:10.201613/GORM.2019.872 \\
\hline
\end{tabular}

How to cite this article: Gonen SE. Kadirogullari P. Seckin KD. Borekci B. Does Acupuncture Have Any Benefit in the IVF Treatment of Couples with Unexplained Infertility; A Prospective Randomized Controlled Trial. Gynecol Obstet Reprod Med 2020;26(1):38-43 anatomic, infectious and other endocrine causes. It occurs in about $9 \%$ of women in reproductive age. In vitro fertilization (IVF) has the best treatment success in infertility (2). However, many IVF cycles do not result in pregnancy and recurrent treatment may require multiple cycles. For this reason, alternative therapies that may increase success rates are being investigated. Acupuncture, a component of traditional Chinese medicine, is particularly a subject of interest in Western countries due to its ease of administration, lack of side effects, and therapeutic effects (3-5). Studies have shown positive results in nausea and vomiting postoperative pain, substance abuse, and general pain syndromes (6-9). Acupuncture has a long history of treating obstetric, gynecological problems and female infertility $(10,11)$.

Acupuncture presumably induces a sympatho-inhibitory effect on uterus and ovaries by elevating the blood flow and thereby enhances implantation $(12,13)$. As a supplementation to standard therapeutic approaches in infertility treatment, performing acupuncture on the day of egg collection and/or embryo transfer is thought to induce the blood flow to embryo $(14,15)$. However, the exact impact of acupuncture on IVF outcomes is still a matter of debate.

In our study, we investigated the effect of acupuncture performed one hour before and one hour after the embryo transfer on pregnancy rates in unexplained infertility patients. 


\section{Material and Method}

This study was designed as an open-label prospective randomized study on unexplained infertility patients who were referred to the infertility outpatient clinic of Atatürk University Faculty of Medicine, Department of Obstetrics and Gynecology between December 2014 and December 2015; in accordance with the revised CONSORT guidelines. Ethical committee approval was obtained from Erzurum Ataturk University (No: B.30.2.ATA.0.01.00/186. 2015/02/06). The study was conducted in accordance with the Declaration of Helsinki. The participants were enrolled in the study consecutively and randomized by a computerized number generator. A total of sixty unexplained infertility patients gave their informed consent to take part in the study who were between 18 -40 years of age. Patients with systemic disorders (neurological, endocrinological, cardiac systemic disease); those with a causal explanation for infertility, women with additional gynecological illnesses, patients on systemic and/or hormonal drugs were excluded from the study.

Semen analysis values of male partners in unexplained infertility couples; the age of female participants; third day of the menstrual cycle serum levels of follicle stimulating hormone (FSH), luteinizing hormone (LH), estradiol, prolactin, thyroid stimulating hormone (TSH), testosterone, progesterone, number of embryos transferred and embryo qualities were recorded. Serum biochemical markers were detected by using commercial kits (Siemens, Germany) and solid-phase enzyme labeled chemiluminescent immunometric assay. Menstrual state, duration of infertility, primary or secondary infertility, previous treatments for infertility were questioned and recorded. None of the patients included in the study had previously received treatment for infertility. The participants in the study were divided into 2 groups using the online randomization software program (GraphPad Software QuickCalcs) according to the simple randomization method. According to the G-Power analysis conducted after the literature review, with $80 \%$ power, the total number of patients to be included in the study was 60 . Sixty patients were reformed into two group: 30 patients to receive IVF treatment with acupuncture performance and 30 cases without acupuncture. Both groups were given the same controlled ovarian hyperstimulation $(\mathrm{COH})$ protocol, and $\beta$-hCG levels were measured 12 days after the embryo transfer in all patients to check for pregnancy.

Before starting the $\mathrm{COH}$ protocol, endometrial thickness was measured by transvaginal ultrasonography and the number of antral follicles were counted. The initial dose of gonadotropin was determined by considering the patient's ovarian reserve, basal hormonal status, and antral follicle count. Starting from $3^{\text {rd }}$ to $5^{\text {th }}$ days of induction serum estradiol levels were measured; follicle sizes and counts were monitored by transvaginal sonography with 1-3 days' intervals. For ovulation induction, r-FSH was initiated (Gonal-F ${ }^{\circledR}$, Serono,
Switzerland and Puregon ${ }^{\circledR}$, Organon, The Netherlands) and on $5^{\text {th }}$ or $6^{\text {th }}$ days of the cycle, $0.25 \mathrm{mg} /$ day Cetrorelix (Cetrotide $^{\circledR}$, Serono, Switzerland) was initiated as an antagonist to prevent premature ovulation. Ovulation trigger was done by using 250 $\mu \mathrm{g}$ recombinant hCG (Ovitrelle ${ }^{\circledR}$, Serono, Italy). The oocyte pick-up (OPU) procedure was performed after 36-48 hours following the triggering of the ovulation.

Symmetrical acupuncture was performed by sterile intradermal needling to Du4, Ren6, Pc6, Sp8, Lr3, Du20, St29 points and one hour prior to embryo transfer and to Du4, Ren6, St36, Sp6, Sp10, Li4 points one hour after transfer. Steel subcutaneous acupuncture needle $0.25 \times 0.25$ was used during this application. After 15 minutes acupuncture needles were removed from the patient's body.

Following acupuncture, all of the embryos were transferred with the same type of embryo transfer catheter (Labotect ${ }^{\circledR}$ embryo transfer catheter set, Germany), after placing the patient in the dorsal lithotomy position, cleansing the vagina and removing the cervical mucus with a sterile swab. After the procedure, patients continued receiving $100 \mathrm{mg}$ progesterone three times a day as luteal phase support, same as they have been doing since the OPU procedure. $\beta$-hCG level was measured 12 days after embryo transfer and if the pregnancy was achieved, progesterone treatment continued until the $12^{\text {th }}$ gestational week.

Data analyses were done by using SPSS 20.0.0 (Statistical Package for Social Sciences) program. Descriptive data were given as mean \pm standard deviation and $\mathrm{n} / \mathrm{N}(\%)$. In the comparison of numerical data, the student-t test was used for comparison between two groups of normal distribution parameters, and the Mann Whitney-U test was used for comparison between two groups of parameters without normal distribution. Chi-square test was used to compare categorical data. $P$ values $<0.05$ were evaluated as statistically significant.

\section{Results}

Seventy-two patients were evaluated, 12 patients were excluded from the study because of not meeting the inclusion criteria. A total of 30 patients who had acupuncture performed were in the study group and 30 patients who did not have acupuncture constituted the control group (Figure 1). While $61.7 \%(n=37)$ of patients had primary infertility, 38.3\% $(n=$ 23) had secondary infertility. After treatment, $40 \%$ of the patients had a positive $\beta$-hCG value $(n=24)$ and $60 \%(n=36)$ negative. One embryo transfer was performed in $48.3 \%(n=29)$ of infertile patients and 2 embryos were transferred in $51.7 \%$ (n $=31$ ). There was no statistically significant difference between the two groups in terms of descriptive characteristics such as age, total progressive motile sperm count (TPMSC), duration of infertility, FSH, LH, testosterone, estradiol, prolactin, TSH, and progesterone $(p>0.05)$. The values of the patients are given in table I in detail. IVF treatment features and protocol patterns were shown in table II. 
Figure 1: Flowchart of the study population

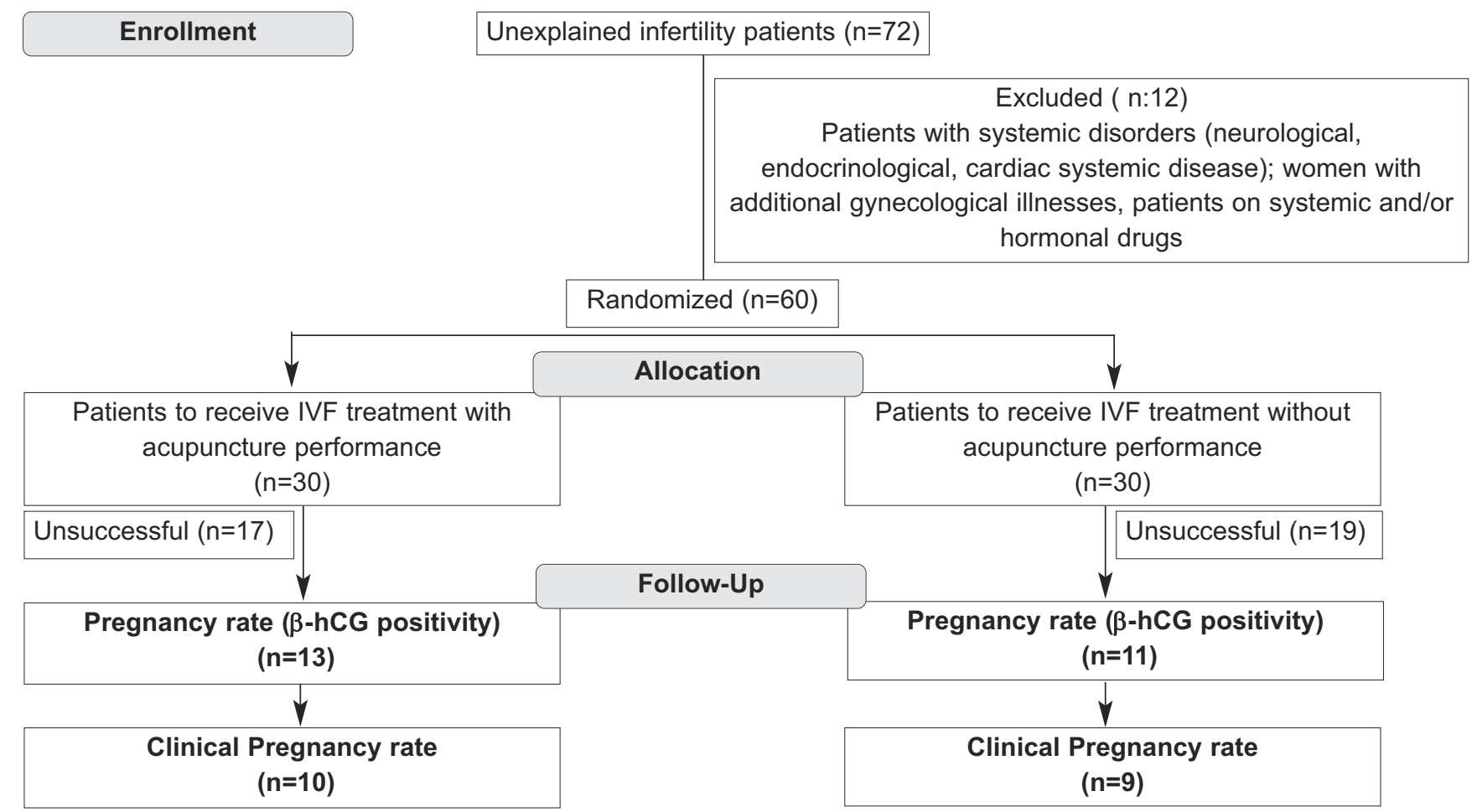

Table I: Characteristic and demographic features of the study participants

\begin{tabular}{llll}
\hline Parameters & Control group $(\mathrm{n}=30)$ & Study group $(\mathrm{n}=30)$ & $p$ \\
\hline Age (years) Mean \pm SD & $31.24 \pm 4.8$ & $32.12 \pm 5.1$ & $>0.05$ \\
BMI $\left(\mathrm{kg} / \mathrm{m}^{2}\right)$ Mean \pm SD & $26.2 \pm 4.1$ & $25.6 \pm 4.7$ & $>0.05$ \\
Duration of infertility (years) Median (Min-Max) & $9(2-21)$ & $1-15)$ & $>(63.3 \%)$ \\
Primary infertility N(\%) & $18(60 \%)$ & $6.07 \pm 1.8$ & $>0.05$ \\
FSH $(\mathrm{mLU} / \mathrm{mL})$ Mean \pm SD & $5.05 \pm 1.2$ & $4.5 \pm 1.8$ & $>0.05$ \\
LH $(\mathrm{mLU} / \mathrm{mL})$ Mean \pm SD & $4.7 \pm 1.6$ & $0.3 \pm 0.1$ & $>0.05$ \\
Testosterone $(\mathrm{ng} / \mathrm{mL})$ Mean \pm SD & $0.3 \pm 0.1$ & $60.5 \pm 28.7$ & $>0.05$ \\
Estradiol $(\mathrm{pg} / \mathrm{mL})$ Mean \pm SD & $57.9 \pm 23.2$ & $11.4 \pm 5.2$ & $>0.05$ \\
Prolactin $(\mathrm{ng} / \mathrm{mL})$ Mean \pm SD & $9.6 \pm 4.6$ & $0.7 \pm 0.5$ & $>0.05$ \\
Progesterone $(\mathrm{pg} / \mathrm{mL})$ Mean \pm SD & $0.6 \pm 0.5$ & $1.8 \pm 1.3$ & $>0.05$ \\
TSH $(\mu U / \mathrm{mL})$ Mean \pm SD & $1.9 \pm 1.4$ & $50 \pm 9.7$ & $>0.05$ \\
TPMSC $(106 / \mathrm{mL})$ Mean \pm SD & $48 \pm 11.8$ & $>0.05$ \\
\hline
\end{tabular}

FSH: Follicle stimulating hormone, LH: Luteinizing hormone, TSH: Thyroid stimulating hormone, TPMSC: Total progressive motile sperm count, ET: Embryo transfer, BMI: Body mass index

Table II: In vitro fertilization treatment features and protocol patterns of the study participants

\begin{tabular}{|c|c|c|c|}
\hline Parameters & Control group $(n=30)$ & Study group $(n=30)$ & $p$ \\
\hline Dose of Gonadotropin (IU) Mean \pm SD & $2250 \pm 475$ & $2350 \pm 625$ & $>0.05$ \\
\hline Days of stimulation Mean \pm SD & $10.2 \pm 1.6$ & $9.9 \pm 1.8$ & $>0.05$ \\
\hline E2 at ovulation induction $(\mathrm{pg} / \mathrm{mL})$ Mean $\pm \mathrm{SD}$ & $1.78 \pm 1,2$ & $1.81 \pm 1.3$ & $>0.05$ \\
\hline Endometrial thickness $(\mathrm{mm})$ Mean $\pm \mathrm{SD}$ & $10.1 \pm 1.9$ & $10.3 \pm 1.8$ & $>0.05$ \\
\hline No. of oocytes retrieved Mean \pm SD & $10.2 \pm 5.2$ & $9.7 \pm 6.4$ & $>0.05$ \\
\hline Number of single ET N(\%) & $14(46.7 \%)$ & $15(50 \%)$ & $>0.05$ \\
\hline
\end{tabular}


All the participants in the study were unexplained infertility patients and when two groups were compared for $\beta$-hCG positivity, there was no significant difference between the groups $(p>0.05)$. Although two groups were not different significantly, the $\beta$-hCG was positive in $43.3 \%$ of the acupuncture patients and in $36.7 \%$ of the patients in the group without acupuncture.

When the study and control groups were investigated, $\beta$-hCG was found to be positive in $48.3 \%$ of the patients receiving a single embryo transfer $(\mathrm{n}=14)$ and it was positive in $32.3 \%(n=10)$ of the cases who had two embryos transferred.

Beta-hCG positivity was observed in $43.3 \%(n=13)$ of 30 patients in the study group while $\beta$-hCG positivity was obtained in $36.7 \%(n=11)$ of 30 patients in the control group. Thus, although there was a higher proportion of biochemical pregnancies in the study group with acupuncture, the difference between the study and control groups was not statistically significant $(p>0.05)$. In terms of clinical pregnancy rates, comparison of the rates was similar between the groups $(33 \%-30 \%)(p>0.05)$. In the study group and control group, pregnancy rates per embryo transfers were $28 \%$ and $24 \%$, respectively.

No complications happened related to acupuncture during the study. After IVF, 1 patient in the study group and 1 woman in the control group, in total 2 patients, developed mild to moderate ovarian hyperstimulation syndrome (OHSS), the patients responded to the treatment and were discharged after complete recovery.

\section{Discussion}

In vitro fertilization is an effective treatment option for many infertile women, families and the community, but new therapies are being sought that may improve pregnancy outcomes due to the relatively low success rate, high cost, and possible confidential safety issues. Acupuncture is an assistive therapy that is often used for many diseases. Acupuncture has been defined as an adjuvant therapy method to improve reproductive outcomes (2).

Until now, the underlying mechanism remains uncertain, despite the numerous studies undertaken to assess the effect of acupuncture on IVF outcomes $(2,3,4,16)$. The effects of acupuncture on IVF or ICSI results depend on various factors such as the choice of type of acupuncture (traditional needling acupuncture, hand acupuncture, ear acupuncture, head acupuncture), the acupuncturist and the effectiveness of the type of acupuncture applied. Acupuncture can facilitate the implantation of embryos by reducing the contractility of the uterus (2). It is also believed that the uterus and ovaries benefit from a general sympatho-inhibitory effect with increased blood flow and thus uterine conditions that support implantation can occur $(12,13)$.
There is heterogeneity in acupuncture treatment and acupuncture point selection in clinical trials. There are currently no standard reference points for generally accepted treatment. It is emphasized that some acupuncture points are not suitable for pregnant women. Therefore, the use of acupuncture in IVF to increase the pregnancy rate extends traditional acupuncture beyond the original field of application $(16,17)$.

In the meta-analysis done by Shen et al, there was no significant difference in the success of acupuncture during embryo transfer (ET), however acupuncture performed 25 minutes before and after ET proved to be relatively beneficial although the difference did not reach statistical significance (18).

Even though recent reviews and meta-analyses have shown that adjuvant acupuncture is not beneficial in terms of IVF results $(19,20)$, a new meta-analysis done by Qian et al. shows that acupuncture might have a beneficial effect on IVF outcomes, especially in the Asian region, and suggests that in order to define a standard acupuncture dose and protocol, further studies are required (2).

A recent meta-analysis by Manheimer et al. covers 16 randomized controlled trials comparing acupuncture performance done one day after ET with sham acupuncture or no adjuvant treatment at all. The addition of one or three performances of acupuncture as an adjuvant to IVF did not elicit a statistically significant advantage (20).

In their study, Zhang et al. compared two groups one of which had acupuncture treatment done on the day of OPU $(\mathrm{OR}=0.85,95 \% \mathrm{Cl} 0.59-1.29, \mathrm{n}=465)$, while the other group received the acupuncture treatment on $\mathrm{ET}$ day $(\mathrm{OR}=1.22,95 \%$ $\mathrm{Cl} 0.87-1.70, \mathrm{n}=2505)$ (21). The difference between the success rates of two groups did not reach statistical significance, thus failed to generate high quality evidence that acupuncture affects IVF success (21).

Westergoard et al. divided IVF and ICSI patients into 3 groups for the study constituting a total of 2 intervention groups and 1 control group. ACU $1(n=95)$ received acupuncture treatment on ET day, patients in ACU $2(n=91)$ had acupuncture done both on ET day and 2 days after and ACU 3 group $(n=87)$ did not receive any kind of acupuncture treatment. Pregnancy rates were compared in all 3 groups and in ACU 1 group, pregnancy rates were higher. Clinical and biochemical pregnancy rates were higher in the ACU 2 group than in the control group ACU 3, but this difference was not statistically significant (22).

In the study conducted by Dieterle et al., 225 infertile patients were allocated into two groups such as 116 were in group 1 in which the patients received traditional Chinese medicine acupuncture and 109 were in group 2 consisting of patients who received placebo acupuncture. Two groups were compared in terms of clinical pregnancy and biochemical 
pregnancies. The patients in Group 1 were found to have dramatically higher $\beta$-hCG positivity, i.e. pregnancy formation, than group 2 (23).

Smith et al. from Australia conducted a study on a total of 228 IVF patients. The patients in the study group were treated with acupuncture on Day 9 of the oocyte stimulation and immediately before and after the embryo transfer, while the second group received non-invasive placebo needling (24). Pregnancy rates, implantation rates and the course of the obtained pregnancies at 18 weeks were investigated. Pregnancy rates were $31 \%$ in the acupuncture group and $23 \%$ in the control group (placebo group). In this case, although the pregnancy rates of the acupuncture group were 1.35 times higher than the placebo group, this difference was not statistically significant. The ongoing $18^{\text {th }}$-week pregnancy rates were higher in the acupuncture group (28\% and $18 \%)$, but this difference was not found statistically significant, either. While there was no significant difference in terms of pregnancy rates among the groups in the study, the therapeutic effect of acupuncture could not be underestimated and another outcome of the study is that acupuncture is safe for women who are scheduled for an embryo transfer (24).

In a study conducted by Paulus et al., 160 patients were randomized into two groups of 80 patients each and while one group received acupuncture treatment 25 minutes before and after transfer of the embryo and the control group was treated with IVF without acupuncture (17). The endpoint was the visualization of fetal sac in the uterus in USG after 6 weeks from the embryo transfer. In the study group who received acupuncture, 34 patients had pregnancy out of 80 patients and in the control group, 21 patients achieved pregnancy out of 80 patients; authors concluded that acupuncture significantly increased the success rate of assisted reproductive technology $(p=0.03)$ (17), Briefly, Cheong et al. (19) reported that acupuncture did not increase IVF success, pregnancy or implantation rates neither statistically nor proportionally. Westergoard et al. (22) concluded that acupuncture was found to be proportionally effective but not statistically significant. Finally, Paulus et al. (17) asserted that acupuncture significantly increased pregnancy success in IVF. In our study, $\beta$-hCG positivity was observed in $43.3 \%(n=13)$ of 30 patients in the study group while $\beta$-hCG positivity was obtained in $36.7 \%(n=11)$ of 30 patients in the control group. Although there was a higher rate of biochemical pregnancy in the acupuncture group, no significant statistical difference was found $(p>0.05)$. Also, clinical pregnancy rates were similar between the groups. The insufficiency of the number of participants and the fact that the acupuncture was performed only before and after embryo transfer did not make statistical significance. Our study is valuable considering that there are only a few studies in Turkish literature, homogeneity of the control and study groups, the acupuncture being performed by the same person, and the ET being performed by the same expert.

\section{Conclusion}

There was no statistically significant effect of acupuncture on pregnancy outcome in our study. This may be due to an insufficient number of patients. In future, in order to increase the accuracy and precision of the studies, acupuncture can start a few days before the embryo transfer and be performed every other day, new needling points can be tested and studies can be done with larger sample sizes.

Acknowledgments: None

Source of funding: None

Conflict of interests: The authors declare that they have no conflict of interests.

Authors Contributions: SEG: Writing, original draft, protocol/project development, data curation. PK: Writing, investigation, review, editing, formal analysis. KDS: Writing, review, editing, formal analysis. BB: Data curation, investigation, protocol/project development.

\section{References}

1. Cramer DW, Walker AM, Schiff I. Statistical methods in evaluating the outcome of infertility therapy. Fertil Steril. 1979;32(1):80-6.

2. Qian Y, Xia XR, Ochin H, Huang C, Gao C, Gao L, et al. Therapeutic effect of acupuncture on the outcomes of in vitro fertilization: a systematic review and meta-analysis. Arch Gynecol Obstet. 2017;295(3):543-58.

3. Qu F, Zhou J, Ren RX. Effects of acupuncture on the outcomes of in vitro fertilization: a systematic review and meta-analysis. J Altern Complement Med. 2012;18(5): 429-39.

4. El-Toukhy T, Khalaf Y. A new study of acupuncture in IVF: pointing in the right direction. Reprod Biomed Online. 2010;21(3):278-9.

5. Hunt KJ, Coelho HF, Wider B, Perry R, Hung SK, Terry $\mathrm{R}$, et al. Complementary and alternative medicine use in England: results from a national survey. Int J Clin Pract. 2010;64(11):1496-502.

6. Dundee JW, Ghaly RG, Fitzpatrick KT, Abram WP, Lynch GA. Acupuncture prophylaxis of cancer chemotherapy-induced sickness. J R Soc Med. 1989;82(5):268-71.

7. Christensen PA, Noreng M, Andersen PE, Nielsen JW. Electroacupuncture and postoperative pain. Br J Anaesth. 1989;62(3):258-62.

8. He D, Berg JE, Hostmark AT. Effects of acupuncture on smoking cessation or reduction for motivated smokers. Prev Med. 1997;26(2):208-14.

9. Shlay JC, Chaloner K, Max MB, Flaws B, Reichelderfer $\mathrm{P}$, Wentworth D, et al. Acupuncture and amitriptyline for pain due to HIV-related peripheral neuropathy: a randomized controlled trial. Terry Beirn Community Programs for Clinical Research on AIDS. JAMA. 1998;280(18):1590-5. 
10. Eisenberg DM, Davis RB, Ettner SL, Appel S, Wilkey S, Van Rompay M, et al. Trends in alternative medicine use in the United States, 1990-1997: results of a follow-up national survey. JAMA. 1998;280(18):156975.

11. Harris P, Rees R. The prevalence of complementary and alternative medicine use among the general population: a systematic review of the literature. Complement Ther Med 2000;8(2):88-96.

12. Stener-Victorin E, Kobayashi R, Kurosawa M. Ovarian blood flow responses to electro-acupuncture stimulation at different frequencies and intensities in anaesthetized rats. Auton Neurosci. 2003;108(1-2):50-6.

13. Stener-Victorin E, Kobayashi R, Watanabe O, Lundeberg T, Kurosawa M. Effect of electro-acupuncture stimulation of different frequencies and intensities on ovarian blood flow in anaesthetized rats with steroid-induced polycystic ovaries. Reprod Biol Endocrinol. 2004;2;16.

14. Stener-Victorin E, Waldenström U, Wikland M, Nilsson L, Hägglund L, Lundeberg T. Electro-acupuncture as a peroperative analgesic method and its effects on implantation rate and neuropeptide Y concentrations in follicular fluid. Hum Reprod. 2003;18(7):1454-60.

15. Humaidan P, Stener-Victorin E. Pain relief during oocyte retrieval with a new short duration electro-acupuncture technique - an alternative to conventional analgesic methods. Hum Reprod. 2004;19(6):1367-72.

16. Craig LB, Rubin LE, Peck JD, Anderson M, Marshall LA, Soules MR. Acupuncture performed before and after embryo transfer a randomized controlled trial. J Reprod Med. 2014;59(5-6):313-20.

17. Paulus WE, Zhang M, Strehler E, El-Danasouri I, Sterzik $\mathrm{K}$. Influence of acupuncture on the pregnancy rate in pa- tients who undergo assisted reproduction therapy. Fertil Steril. 2002;77(4):721-4.

18. Shen C, Wu M, Shu D, Zhao X, Gao Y. The role of acupuncture in in vitro fertilization: a systematic review and meta-analysis. Gynecol Obstet Invest. 2015;79(1): $1-12$.

19. Cheong YC, Dix S, Hung Yu Ng E, Ledger WL, Farquhar C. Acupuncture and assisted reproductive technology. Cochrane Database Syst Rev. 2013;7:CD006920.

20. Manheimer E, van der Windt D, Cheng K, Stafford K, Liu $\mathrm{J}$, Tierney $\mathrm{J}$, et al. The effects of acupuncture on rates of clinical pregnancy among women undergoing in vitro fertilization: a systematic review and meta-analysis. Hum Reprod Update. 2013;19(6):696-713.

21. Zhang M, Huang G, Lu F, El-Danasouri I, Sterzik K: Effect of acupuncture on rate of pregnancy among women undergoing embryo transfer: a prospective randomized placebo controlled trial. Zhongguo Zhen Jiu. 2003;23:3-5.

22. Westergaard LG, Mao Q, Krogslund M, Sandrini S, Lenz S, Grinsted J. Acupuncture on the day of embryo transfer significantly improves the reproductive outcome in infertile women: a prospective, randomized trial. Fertil Steril. 2006;85(5):1341-6.

23. Dieterle S, Ying G, Hatzmann W, Neuer A. Effect of acupuncture on the outcome of in vitro fertilization and intracytoplasmic sperm injection: a randomized, prospective, controlled clinical study. Fertil Steril. 2006;85(5): 1347-51.

24. Smith C, Coyle PM, Norman RJ. Influence of acupuncture stimulation on pregnancy rates for women undergoing embryo transfer. Fertil Steril. 2006; 85(5):1352-8. 